\title{
Modeling of Technical Training of Discus Throwers in the Period of Significant Changes of Their Mass-inertia Characteristics
}

\author{
Mikhail P. Shestakov \\ Research Institute of Sport Problems, Russian State University of Physical Education, Moscow, Russia
}

\begin{abstract}
The use of artificial neural networks for simulation of the control of an anthropomorphic mechanism when its mass-inertia characteristics are changing is considered in this paper. It is shown in theory that the change in mass-inertia characteristics of the model does not have a linear relationship with the output parameter but a spurt-like change in the pattern of the output function after a certain threshold. The data of the model experiment served as a basis for a full-scale test with discus throwers who had different height-weight parameters during a year of study. Specific traits in the technique of standing discus throwing are shown, and their dependence on the changes of anthropometric characteristics of sportsmen. $J$ Physiol Anthropol Appl Human Sci 24(4): 367-370, 2005 http://www.jstage.jst.go.jp/browse/jpa

[DOI: 10.2114/jpa.24.367]
\end{abstract}

Keywords: artificial neural network, mass-inertia characteristics, biomechanics, sportsman

\section{Introduction}

The treatment of huge data files resulting from mass anthropological investigations implies the problem of choices of an appropriate mathematical approach. The presence of incomplete data, multifactorial objects, values that are simply missing in the protocols, and absence of strict rules make the process of statistical analysis very difficult. At present, some of the tasks with the above-mentioned discrepancies are solved with a neural networks approach. One of the major characteristics of this approach is the possibility of selfinstruction (self-regulation) of a mathematical tool according to the specific traits of that database which needs to be analysed.

The idea of neural networks emerged as a result of attempts to simulate the behavior of living things sensitive to external influences and able to learn from their own experience (Lippman, 1987). Ideas of this kind, appearing at the interface of different disciplines, are typical of modern science.

Artificial neural networks can change their behavior depending on the external environment. After input signals are presented (possibly, with the required outputs) they adjust themselves to produce the reaction needed (Rumelhart et al., 1986). The response of the network after self-adjustment can be to some extent non-sensitive to small changes of the input signals. This inherent internal ability to see the image through the noise and distortion is very important for the recognition of images in the real world (Hopfield, 1982).

From the mathematical point of view, a neural network is a multilayer structure consisting of relatively simple processing elements of one type - neurons. Neurons interconnected with a complex topology of associations are grouped into layers (usually two or three). Among those, input and output layers can be separated. In neural networks used for prediction, the input neurons perceive information about the situational parameters, while the output layer is signaling about possible reaction to this situation.

Usually neural networks are used for qualification tasks, taxonomy, prediction and modeling non-linear relationships (non-linear regression).

Recently, neural networks have started to be used for the socalled neurobionic approach suggested by Pospelov (Averkin et al., 1992) in the context of a new scientific discipline "biocyberogogics" (Shestakov, 2000), which was used as a basis for the development of experimental and simulation studies.

The purpose of the present study is a simulation of the process of the learning of a highly coordinated motor action under the changes of mass-inertia characteristics in humans.

\section{Modeling Experiment}

For this experiment with the use of adjusted neural networks let us take a simplistic model consisting of a pivot with a fixed end, which is controlled by a neural network via sending corresponding signals to two elastic traction-antagonists (Fig. 1). Evidently, the values of the control moment (Mr) will be influenced by the values of length (L) and mass $(\mathrm{m})$ of the pivot, which appear in the equation. In Table 1 the data of the model experiment with the adjusted neural network are given. 
Table 1 The results of the model experiment in the prediction changes of the instructed neural network (s) at different values of the pivot length and mass

\begin{tabular}{c|c|c|c|c|c|c|c|c|c}
\hline & \multicolumn{10}{c}{ Increase of value } \\
\cline { 2 - 15 } & $0 \%$ & $0.5 \%$ & $1 \%$ & $1.5 \%$ & $2 \%$ & $2.5 \%$ & $3 \%$ & $3.5 \%$ & $4 \%$ \\
\hline Pivot length L & 0.8 & 0.8 & 0.8 & 1.3 & 1.8 & 5.0 & 6.1 & 7.9 & 12.1 \\
\hline Pivot mass m & 0.8 & 0.8 & 0.8 & 0.8 & 10 & 1.2 & 4.3 & 4.9 & 8.3 \\
\hline
\end{tabular}
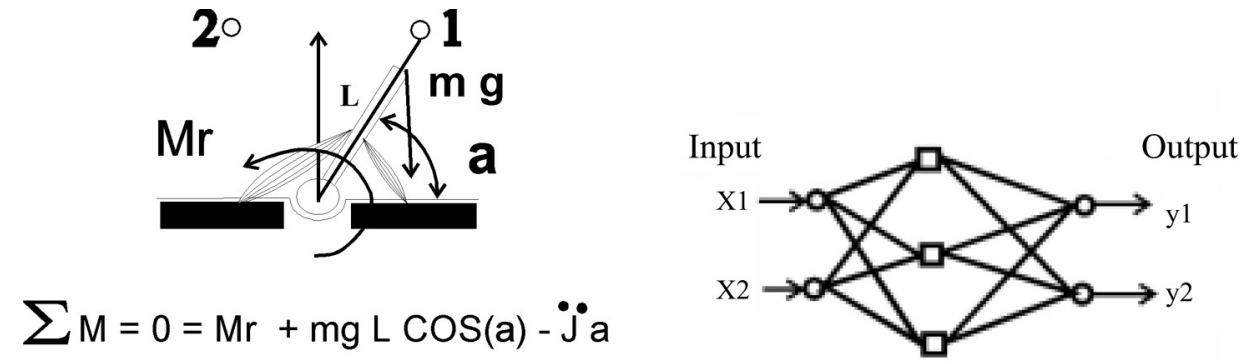

Fig. 1 Diagram of the pivot's movements control from position 1 to position 2 with the help of the adjusted neural network. Elements: pivot, hinge, base, control moment. The length of the pivot is L; mass is $\mathrm{m}$; inertia moment is $\mathrm{J}$; angle of inclination is a, and $\mathrm{Mr}=\mathrm{f}(\mathrm{i}, \mathrm{a}$,á), that is control moment, depending on length (force), speed (viscosity and Hill's law), and activation level i. Neural network: X1 is type of motive program, $\mathrm{X} 2$ is intensity of performing a movement, $\mathrm{y} 1$ is "muscular activity" of the agonist, and y2 is "muscular activity" of the antagonist.

After the network is instructed in the recognition of image "2" (the goal for the pivot's shift from position "1" to position "2" via "muscular activity" of the agonist and antagonist), the network input X1 (a version of the motive program) has undergone some distortion. The distortion consisted of the changes in length $(\mathrm{L})$ and mass $(\mathrm{m})$ of the pivot by a certain value (\%). The results of the simulation show that an increase in length by $2.5 \%$ or more, and a pivot mass by $3 \%$ bring a significant deterioration of the network's predictions (s), which reflects the disturbances in the agonist-antagonist coordination.

The analysis show that there is a certain threshold after which the changes in one of the input values result in the changes of the output values with a significant loss in the quality of the network's functioning. In fact, for normal network functioning it is necessary to go through a new procedure of learning (instruction) but, naturally, on the basis of an already obtained matrix. Thus, it is shown that the change in mass-inertia characteristics of the model does not bring a linear relationship with the output parameter but a spurt-like change in the pattern of the output function after a certain threshold. This can be defined as the PRINCIPLE of INDIVIDUALIZATION.

The organization of the process of long technical training is affected to a great extent by biological changes in the growing body of a sportsman. The most evident changes refer to the growth of body mass and body proportions.

Parallel to skeletal and muscular growth, physical strength is increasing. At first it lags behind muscle development. It reflects a common pattern when the structure is formed before the corresponding function. There is a sequence developing in time: muscle growth - muscle strength - coordination. Hence, accurate functioning can be absent at the beginning and be gradually developed. This is particularly important for the coordinated activity of different groups of muscles.

Consequently, under a constant level of special physical training, as in our case, changes in mass geometry can result in both positive and negative influences on the efficacy of the biomechanics of motor activity.

The discus speed is a result of a combination of forces of separate body segments-legs, trunk, and arm. In theory there are two systems of interaction of body parts when the speed of the end unit is maximal. The first one is characterized by organization of the movement in such a way that maximal speed in different sections is produced simultaneously. The other way implies a consequent acceleration in the segments from bottom to top, that is, each of the consequent body parts starts moving when the speed of the previous one reaches its maximum. In practice, and according to experimental data, sportsmen perform movements using the second technique. The analysis shows that for sportsmen with a different qualification the strategy of movement organization is different.

We can separate the following biomechanisms in discus throwing (Seluyanov et al., 1995), which, by definition, can achieve the goal task operating independently from each other (for simplification only the final part of throwing will be considered):

- Biomechanism of rotation around pivot;

- Biomechanism of legs unbending;

- Biomechanism of whipping.

The first two biomechanisms are not difficult to define. The special role of the biomechanism of whipping should be noted. It is this mechanism that gives speed to the apparatus in all kinds of throwing. The point is that sportsmen induce successive acceleration and inhibition of body parts from bottom to top. Such actions allow the use of biomechanical 
muscle properties, with elasticity as one of the main ones. The acceleration of movement of the proximal end of the section, as opposed to the distal end, permits the stretching of the muscles performing at the proximal end of the joint. The energy of elastic deformation is accumulated in these muscles. Part of this energy at successive joint bending is spent on the acceleration of the distal end of the section. However, the described mechanism operates effectively only in combination with other biomechanisms.

\section{Methods}

To evaluate the contribution of each biomechanism in the process of discus throwing, the analysis of throwing from a standing position performed by sportsmen of different ages was carried out in the Research Institute of Sport Problems at the Russian State University of Physical Education.

In the course of the experiment, the dynamic characteristics of vertical and horizontal components of a basic reaction were recorded using the tensodynamographic platform PD-3. Kinematic parameters of throwing performance were evaluated by videotaping. Simultaneously, electromyography of activity in four muscles was recorded: the right musculus gastrocnemius, the left musculus biceps femoris, the left musculus obliquus abdominis, and the right musculus pectoralis major. The EMG-signals were recorded with a "Medicor" Electromyographer, skin electrodes at a distance of $20 \mathrm{~mm}$, and input of on-line computer data. Further analysis was performed with special software.

57 discus throwers, 13-to-19-year-old males, took part in the experiment.

\section{Results and Discussion}

The analysis of the results show that there are typical patterns of the registered parameters for each age group and qualification level of a sportsman.

At the stage of initial specialization sportsmen perform the throwing primarily at the expense of movements of the shoulder girdle.

The stage of more advanced specialization is characterized by diverse actions of the lower extremities and upper shoulder girdle.

At the stage of athletic perfection the throwers perform the movement in the following order: after the right knee-joint begins to unbend, the right hip joint starts unbending, then the oblique abdomen muscles take part in the movement, and finally the muscles of the shoulder joint of the throwing arm come into play.

The results of reprocessing of the experimental data permit us to evaluate the contribution of biomechanisms taking part in throwing from a standing position in groups of sportsmen of different ages. The contribution of each biomechanism was estimated by the analysis of the speed parameter in the system "thrower-discus" in separate phases of the throwing performance.

Sportsmen of lower qualification, who were still mastering the throwing technique, used primarily the shoulder girdle.

Sportsmen of high qualification use to a greater extent the mechanism of whipping when showing outstanding results.

The group of 14-to-15-year-old sportsmen is of special interest as a significant intragroup variability in the contribution of the whipping biomechanism was shown in this group. It was divided into two subgroups according to the rates of annual increase in growth parameters (mass-inertia characteristics (Table 2)).

As can be seen from the table, there is a definite decrease in the contribution of the whipping biomechanism depending on the changes of growth parameters in sportsmen.

The situation described has been modeled with a neural network approach. As input parameters, contributions (\%) of each biomechanism and mass-inertia characteristics were used; as ouput, the speed of disk offset was produced by a neural network.

The analysis of network functioning has shown that a gradual increase of a sportsman's mass-inertia characteristics, other conditions being equal, gives a certain increase in the output parameter. However, if $\Delta$ mass-inertia characteristics (length parameters) are more than $7.5 \mathrm{~cm}$ per year, there is a sharp decrease in the values of the output parameter.

Further simulation has shown that the optimal training technique for sportsmen with significant mass-inertia characteristics changes includes the use of technical means orientated to separate training of a rotation biomechanism and

Table 2 The results of a real experiment with 14-to-15-year-old discus throwers

\begin{tabular}{llll}
\hline $\begin{array}{l}\text { Contribution of } \\
\text { biomechanisms, } \%\end{array}$ & $\begin{array}{l}\text { Biomechanism of } \\
\text { rotation around } \\
\text { pivot }\end{array}$ & $\begin{array}{l}\text { Biomechanism } \\
\text { of legs } \\
\text { unbending }\end{array}$ & $\begin{array}{l}\text { Biomechanism } \\
\text { of whipping }\end{array}$ \\
\hline $\begin{array}{l}\text { Group A } \\
\text { mass-inertia } \\
\text { characteristics }<5 \mathrm{~cm} / \text { year }\end{array}$ & 30 & 30 & 40 \\
\hline $\begin{array}{l}\text { Group B } \\
\text { chass-inertia } \\
\text { characteristics }>7.5 \mathrm{~cm} / \text { year }\end{array}$ & 50 & 25 & 25 \\
\hline
\end{tabular}


leg unbending biomechanism. As a result, a special training program was prepared for young sportsmen with the abovedescribed differences in their morphofunctional development.

\section{References}

Averkin AN, Gaase-Rappoport MG, Pospelov DA (eds.) (1992) Artificial intelligence. Dictionary of Definitions. Radio i svyaz, Mowcow, 1-256 [In Russian]

Carr J (1970) The discus throw technique. Athletics Coach 4: 5-9

Hopfield JJ (1982) Neural network and physical systems with emergent collective computational abilities. Proc Nat Sci USA 79: 2554-2558

Lippman RP (1987) An introduction to computing with neural nets. IEEE ASSP Magazine (Apr): 4-22

Rumelhart DE, Hinton GE, Williams RJ (1986) Learning representations by back-propagation errors. Nature 323:
$533-536$

Seluyanov VN, Shalmanov AnA, Annenkov KA, Gregorenko AV (1995) Biomechanisms as the basis of biomechanics of human movements in sport. Theory and Practice of Physical Education 7: 6-10 [In Russian]

Shestakov M (2000) Artificial Intelligence in Sport Science of the 21 st Century. Theory and Practice of Physical Education 7: 8-14 [In Russian]

Received: March 29, 2005

Accepted: April 6, 2005

Correspondence to: Professor Mikhail P. Shestakov, Research Institute of Sport Problems, Russian State University of Physical Education, Syrenevyi blvd 4, Moscow105122, Russia

Phone: +7-095-166-4892

Fax: +7-095-166-4892

e-mail: shtv@infosport.ru 